\title{
The combinatorics of LCD codes: Linear Programming bound and orthogonal matrices
}

\author{
Steven T. Dougherty* $\quad$ Jon-Lark Kim ${ }^{\dagger} \quad$ Buket Ozkaya ${ }^{\ddagger} \quad$ Lin Sok $\S$ \\ Patrick Soléđ
}

\begin{abstract}
Linear Complementary Dual codes (LCD) are binary linear codes that meet their dual trivially. We construct LCD codes using orthogonal matrices, self-dual codes, combinatorial designs and Gray map from codes over the family of rings $R_{k}$. We give a linear programming bound on the largest size of an LCD code of given length and minimum distance. We make a table of lower bounds for this combinatorial function for modest values of the parameters.
\end{abstract}

Key Words: LCD (linear codes with complementary dual) codes, Self-dual codes, Linear programming bound.

MSC (2010) 94B 05, 20H 30

\footnotetext{
*Department of Mathematics, University of Scranton, Scranton, PA 18510, USA. Email: prof.steven.dougherty@gmail.com

$\dagger^{\dagger}$ Department of Mathematics, Sogang University, Seoul 121-742, South Korea. Email: jlkim@sogang.ac.kr

${ }^{\ddagger}$ CNRS/LTCI, UMR 5141, Télécom-ParisTech, 46 rue Barrault 75634 Paris cedex 13, France. Email: ozkaya@enst.fr

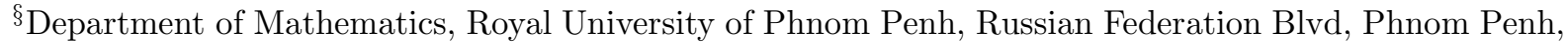
Cambodia. E-mail:sok.lin@rupp.edu.kh

${ }^{\top}$ CNRS/LTCI, UMR 5141, Télécom-ParisTech, 46 rue Barrault 75634 Paris cedex 13, France. Email: sole@enst.fr
} 


\section{Introduction}

In this paper, we study linear codes with complementary duals, which we refer to as LCD codes. These codes were introduced by Massey in [9] and give an optimum linear coding solution for the two user binary adder channel. They are also used in counter measures to passive and active side channel analyses on embedded cryto-systems, see [3] for a detailed description.

The main result is a linear programming bound on the largest size of an LCD code of given length and minimum distance. We show by numerical examples that this bound is, in general, sharper than the standard linear programming bound on the size of codes of given length and distance. We also give a combinatorial construction of LCD codes based on orthogonal matrices, which are essentially equivalent to systematic generator matrices of self-dual codes. They also enjoy a pseudo-random construction due to their multiplicative groups structure. It is important to note that a single self-dual code, or, equivalently a single orthogonal matrix give rise to several LCD codes. We sketch another construction by codes over rings and Gray maps, and a construction based on symmetric designs. A table of lower bounds on the largest size of an LCD code of given length and minimum distance is built based on the orthogonal matrix construction.

The material is organized as follows. Section 2 contains some constructions of LCD codes over rings that impact LCD codes over fields. Section 3 introduces and studies two combinatorial functions related to LCD codes. Section 4 derives the linear programming bound and provide a comparative numerical table with the standard linear programming bound. Section 5 contains the various constructions from rings, matrices and block designs. A last section concludes the paper and paves the way to new research.

\section{Preliminaries}

In this work, we shall be largely concerned with codes over finite fields. However, we shall use codes over rings together with a linear Gray map to construct LCD codes. Hence we shall make the definitions in a general setting. A code $C$ of length $n$ over a ring $R$ is a subset of $R^{n}$. All rings in this paper are assumed to be commutative rings with unity. If the code is a submodule then the code is said to be linear. Attached to the ambient space is the standard inner-product, namely $[\mathbf{v}, \mathbf{w}]=\sum v_{i} w_{i}$. The orthogonal is defined by $C^{\perp}=\left\{\mathbf{v} \in R^{n} \mid[\mathbf{v}, \mathbf{w}]=0, \forall \mathbf{w} \in C\right\}$. If $R$ is a Frobenius ring then we have that $|C|\left|C^{\perp}\right|=|R|^{n}$. For codes over finite fields we have $\operatorname{dim}(C)+\operatorname{dim}\left(C^{\perp}\right)=n$. 
A linear code with complementary code (LCD) is a linear code $C$ satisfying $C \cap C^{\perp}=\{0\}$. Any code over a field is equivalent to a code generated by a matrix of the form $\left(I_{k} \mid A\right)$ where $I_{k}$ denotes the $k$ by $k$ identity matrix. For codes over rings, this is not the case so we shall talk about generating vectors instead in the following lemmas.

Lemma 2.1 Let $\mathrm{v}_{1}, \mathrm{v}_{2}, \ldots, \mathrm{v}_{k}$ be a vectors over a commutative ring of characteristic 2 such that $\left[\mathbf{v}_{i}, \mathbf{v}_{i}\right]=1$ for each $i$ and $\left[\mathbf{v}_{i}, \mathbf{v}_{j}\right]=0$ for $i \neq j$. Then $C=\left\langle\mathbf{v}_{1}, \mathbf{v}_{2}, \ldots, \mathbf{v}_{k}\right\rangle$ is an LCD code.

Proof. Any vector in $C$ is of the form $\mathbf{w}=\sum_{i \in A} \mathbf{v}_{i}$. Then let $j \in A$, it follows that $\left[\mathbf{v}_{j}, \mathbf{w}\right]=1$. Hence $\mathbf{w} \notin C^{\perp}$. This gives that no non-trivial element in $C$ is also in $C^{\perp}$ and hence their intersection is trivial.

Applying this lemma to codes over fields we have the following.

Corollary 2.2 Let $G$ be a generator matrix for a code over a finite field. If $G G^{T}=I_{n}$ then $G$ generates an LCD code.

More generally for codes over fields this leads to the following.

Corollary 2.3 Let $G$ be a generator matrix for a code over a field. Then $\operatorname{det}\left(G G^{T}\right) \neq 0$ if and only if $G$ generates an LCD code.

Lemma 2.4 Let $\mathrm{v}_{1}, \mathrm{v}_{2}, \ldots, \mathrm{v}_{k}$ be a set of vectors over a ring of characteristic 2 such that $\left[\mathbf{v}_{i}, \mathbf{v}_{i}\right]=0$ and $\left[\mathbf{v}_{i}, \mathbf{v}_{j}\right]=1$ if $i \neq j$. Then $C=\left\langle\mathbf{v}_{1}, \mathbf{v}_{2}, \ldots, \mathbf{v}_{k}\right\rangle$ is LCD if and only if $k$ is even.

Proof. Assume $k$ is even. Consider the vector $\mathbf{w}=\sum_{i \in A} \mathbf{v}_{i}$.

If $|A|$ is even take $j \in A$. Then $\left[\mathbf{w}, \mathbf{v}_{j}\right]=1$. If $|A|$ is odd take $j \notin A$ then $\left[\mathbf{w}, \mathbf{v}_{j}\right]=1$. In either case, no linear combination of the generators can be in the orthogonal. Hence the code is LCD.

Assume $k$ is odd. Then $\left[\sum_{i=1}^{k} \mathbf{v}_{i}, \mathbf{v}_{j}\right]=0$ for any $j$. Hence $\sum_{i=1}^{k} \mathbf{v}_{i} \in C \cap C^{\perp}$ and the code is not LCD.

Let $J_{n}$ denote the all one $n$ by $n$ matrix. Considering this lemma as applied to codes over fields we have the following.

Corollary 2.5 Let $G$ be a generator matrix for a code over a finite field. If $G G^{T}=J_{n}-I_{n}$, $n$ even, then $G$ generates an LCD code. 


\section{Elementary Bounds}

In this section, we are only concerned with codes over the binary field.

\subsection{Fixed $n$ and $k$.}

Let $\mathbf{L C D}[n, k]:=\max \{d \mid$ there exists a binary $[n, k, d] \mathbf{L C D}$ code $\}$.

Lemma 3.1 For $n$ and $k$ integers greater than $0, \boldsymbol{L} \boldsymbol{C D}[n+1, k] \geq \boldsymbol{L} \boldsymbol{C D}[n, k]$.

Proof. Let $G$ be a generator matrix of an $[n, k, d]$ LCD code $C$. Then $G G^{T}$ is invertible since $C$ is LCD. Let $\bar{G}$ be the matrix obtained from $G$ by adding the zero column 0 to the right end of $G$, that is, $\bar{G}=G \mathbf{0}$. $\bar{G}(\bar{G})^{T}=G G^{T}$ is invertible. Hence $\bar{G}$ generates an $[n+1, k, d] \mathbf{L C D}$ code. Therefore $\mathbf{L C D}[n+1, k] \geq \mathbf{L C D}[n, k]$.

Proposition 3.2 (i) If $n$ is odd, then $\boldsymbol{L} \boldsymbol{C D}[n, 1]=n$ and $\boldsymbol{L} \boldsymbol{C D}[n, n-1]=2$.

(ii) If $n$ is even, then $\boldsymbol{L} \boldsymbol{C D}[n, 1]=n-1$ and $\boldsymbol{L} \boldsymbol{C D}[n, n-1]=1$.

Proof. (i) It is clear that the repetition $[n, 1, n]$ code and its dual are LCD and have the highest minimum distances.

(ii) If $n$ is even, the repetition $[n, 1, n]$ code is not LCD since its dual contains the all-one vector. It is easy to see that the code $C$ with generator matrix $\left[\begin{array}{llll}0 & 1 & 1 & \ldots\end{array}\right]$ is $\mathbf{L C D}$. Thus $\mathbf{L C D}[n, 1]=n-1$. The dual $C^{\perp}$ of $C$ is LCD with minimum distance 1 . If $\operatorname{LCD}[n, n-1]=2$, then the corresponding code is the even $[n, n-1,2]$ code which is not LCD since the all-one vector belongs to the even code and the repetition code of length $n$. Thus $\operatorname{LCD}[n, n-1]=1$.

Lemma 3.3 The following hold.

(i) $\boldsymbol{L} \boldsymbol{C D}[n m, k l] \geq \boldsymbol{L} \boldsymbol{C D}[n, k] \boldsymbol{L} \boldsymbol{C D}[m, l]$.

(ii) $\boldsymbol{L} \boldsymbol{C D}[n+m, k+l] \geq \min \{\boldsymbol{L} \boldsymbol{C D}[n, k], \boldsymbol{L} \boldsymbol{C D}[m, l]\}$.

Proof. (i) Let $G_{1}$ be a generator matrix of an $\left[n, k, d_{1}\right]$ LCD code $C_{1}$ and $G_{2}$ a generator matrix of an $\left[m, l, d_{2}\right]$ LCD code $C_{2}$. Consider the direct product of $C_{1}$ and $C_{2}$, denoted by $C_{1} \otimes C_{2}$, which has parameters $\left[n m, k l, d_{1} d_{2}\right]$ (see [8, Ch. 8]). The generator matrix of $C_{1} \otimes C_{2}$ is the Kronecker product of $G_{1}$ and $G_{2}$, denoted by $G_{1} \otimes G_{2}$. We need to show that $C_{1} \otimes C_{2}$ is LCD. It suffices to show that $\left(G_{1} \otimes G_{2}\right)\left(G_{1} \otimes G_{2}\right)^{T}$ is invertible. Note that $\left(G_{1} \otimes G_{2}\right)\left(G_{1} \otimes G_{2}\right)^{T}=\left(G_{1} \otimes G_{2}\right)\left(G_{1}^{T} \otimes G_{2}^{T}\right)=$ 
$\left(G_{1} G_{1}^{T} \otimes G_{2} G_{2}^{T}\right)$. Since $G_{i} G_{i}^{T}$ (for $\left.i=1,2\right)$ is invertible, $\left(G_{1} G_{1}^{T} \otimes G_{2} G_{2}^{T}\right.$ ) is invertible because $\left(G_{1} G_{1}^{T} \otimes G_{2} G_{2}^{T}\right)\left(\left(G_{1} G_{1}^{T}\right)^{-1} \otimes\left(G_{2} G_{2}^{T}\right)^{-1}\right)=I_{k} \otimes I_{l}=I_{k l}$, where $I_{a}$ is the identity matrix of order $a$.

(ii) It is known [3] that the direct sum of $C_{1} \oplus C_{2}$ of LCD codes $C_{1}, C_{2}$ of parameters $\left[n, k, d_{1}\right]$ and $\left[m, l, d_{2}\right]$ respectively is also an LCD code of parameters $\left[n+m, k+l, \min \left\{d_{1}, d_{2}\right\}\right]$. Hence $\mathbf{L C D}[n+m, k+l] \geq \min \{\mathbf{L C D}[n, k], \mathbf{L C D}[m, l]\}$ follows.

\subsection{LCD $[n, k]$ for small $n, k$}

We have a partial result on $\operatorname{LCD}[n, 2]$ for $n \geq 3$.

Theorem 3.4 We have the following:

(i) $\mathbf{L C D}[3,2]=2$

(ii) $\boldsymbol{L} \boldsymbol{C D}[4,2]=2$

(iii) $\mathbf{L C D}[5,2]=2$

(iv) $\boldsymbol{L} \boldsymbol{C D}[6,2]=3$

(v) $\boldsymbol{L C D}[7,4]=2$

Proof.

1. Choose the even code of length 3 with generator matrix $G=\left[\begin{array}{lll}1 & 1 & 0 \\ 1 & 0 & 1\end{array}\right]$. It is LCD and has minimum distance $d=2$. There is no $[3,2,3]$ code. Thus $\mathbf{L C D}[3,2]=2$.

2. There is $[4,2,2]$ LCD namely the $B K L C(G F(2), 4,2)$ provided by Magma. Hence $\mathbf{L C D}[4,2]=2$.

3. Since there is no nontrivial MDS binary code, there is no $[5,2,4]$ code. There are two $[5,2,3]$ codes up to equivalence. They have generator matrices such as

$$
\left[\begin{array}{lllll}
1 & 0 & 1 & 1 & 0 \\
0 & 1 & 1 & 1 & 1
\end{array}\right] \text { and }\left[\begin{array}{lllll}
1 & 0 & 1 & 1 & 0 \\
0 & 1 & 0 & 1 & 1
\end{array}\right] \text {. }
$$

None of them are LCD. Thus $\operatorname{LCD}[5,2] \leq 2$, and so $\operatorname{LCD}[5,2]=2$ by Lemma 3.1 and (i) above. 
4. Note that the repetition $[3,1,3]$ code is LCD. Thus by (ii) of Lemma 3.3 , we have $\mathbf{L C D}[6,2] \geq 3$. If $\mathbf{L C D}[6,2]=4$, then there is a unique $[6,2,4]$ code $C$ whose generator matrix can be arranged up to equivalence as

$$
G=\left[\begin{array}{llllll}
1 & 0 & 1 & 1 & 1 & 0 \\
0 & 1 & 0 & 1 & 1 & 1
\end{array}\right] .
$$

Since $G G^{T}$ is a zero matrix (hence noninvertible), $C$ is not LCD. Thus $\mathbf{L C D}[6,2]=3$.

5. There is a $[7,2,4]$ code whose generator matrix is given by

$$
G=\left[\begin{array}{lllllll}
1 & 1 & 1 & 1 & 0 & 0 & 0 \\
0 & 0 & 0 & 1 & 1 & 1 & 1
\end{array}\right]
$$

It is known that an optimal $[7,2]$ code has $d=4[5]$. Therefore $\operatorname{LCD}[7,2]=4$.

\section{3 $\quad$ Fixed $n$ and $d$}

We introduce the combinatorial function $\operatorname{LCK}[n, d]:=\max \{k \mid$ there exists a binary $[n, k, d] \mathbf{L C}]$ From first principles, we see that $\mathbf{L C K}[n, d] \leq \log _{2} A(n, d)$. Some values of that function for small $d$ are easy to find.

Proposition 3.5 For all $n \geq 1$, we have $\mathbf{L C K}[n, 1]=n$.

Proof. The complete code with dual the null code is LCD. The result follows.

Proposition 3.6 If $n$ is odd then $\mathbf{L C K}[n, n]=1$ and $\mathbf{L C K}[n, 2]=n-1$. If $n$ is even then $\mathbf{L C K}[n, n]=0$, and $\mathbf{L C K}[n, 2]=n-2$.

Proof. The repetition code of odd length is $L C D$ and optimal. Its dual is as well. The first assertion follows. On the other hand the repetition code of even length is unique with their parameters and self-orthogonal, hence not LCD. By adding an extra zero/one coordinate to the dual of the repetition code in length $n-1$ we obtain an LCD code of parameters $[n, n-2,2]$.

Proposition 3.7 For all integers $m>1$ we have

- $\boldsymbol{L} \boldsymbol{C K}\left[2^{m}-1,3\right]<2^{m}-m-1$

- $\boldsymbol{L} \boldsymbol{C K}\left[2^{m}-1,2^{m-1}\right]<m$. 
Proof. The Hamming code and its dual the Simplex code are not LCD since the Simplex code is self-orthogonal. Further these codes are unique with their parameters. This is immediate for the Hamming code. Note that the Simplex code is unique as meeting the Plotkin bound [8, Th. 11(a)], hence one-weight, hence characterized by [2].

Proposition 3.8 We have $\mathbf{L C K}[24,8]<12$, and $\mathbf{L C K}[23,7]<11$.

Proof. The extended Golay code is unique [16, Th. 104] and self-dual. Puncturing once yields another unique code [16, Th. 104] which contains its dual.

Let $g(k, d)=\sum_{i=0}^{k-1}\left\lceil\frac{d}{2^{i}}\right\rceil$ denote the RHS of the Griesmer bound.

Proposition 3.9 If $d$ is a multiple of four, then $\mathbf{L C K}[g(k, d), d]<k$.

Proof. By Theorem 1 of [17], if a code $C$ meets the Griesmer bound with a minimum distance multiple of 4 , then all its weight are multiples of 4 . It is then immediate by the parallelogram identity $[8,(12)$ p.9] that $C$ is self-orthogonal.

\section{Linear Programming bound}

Let $C$ denote a binary linear $[n, k]$ code and $A_{i}$ its weight distribution. Let $B_{i}$ denote the weight distribution of its dual code $C^{\perp}$. Let $P_{i}(x)$ be the Krawtchouk polynomial of degree $i$ given by the following generating function:

$$
\sum_{i=0}^{n} P_{i}(x) z^{i}=(1+z)^{n-x}(1-z)^{x} .
$$

If $M$ is a square matrix of order $N_{R}$ by $N_{C}$ and $x, h$ are column vectors of length $N_{R}$, and $N_{C}$ respectively, we denote by $U(M, h)$ the maximum of $\sum_{j=1}^{N_{C}} x_{i}$ for nonnegative rationals $x_{i}$ subjected to the $N_{R}$ linear constraints $M x \leq h$. We need the following auxiliary matrices:

- $P=\left(P_{j}(i)\right)$;

- $\Delta$ the matrix with entries $\Delta_{i, j}=\left(\begin{array}{l}n \\ i\end{array}\right)$ for all $1 \leq j \leq n$;

- $I_{m}$ the identity matrix of order $m$. 
If $C$ is $\mathbf{L C D}$ then for all $n \geq i \geq 1$ we have from the definition of $L C D$ codes that

$$
A_{i}+B_{i} \leq\left(\begin{array}{c}
n \\
i
\end{array}\right)
$$

a vector of weight being in either $C$ or its dual but not in both.

Now by the MacWilliams formula we know that

$$
B_{i}=2^{-k} \sum_{j=0}^{n} A_{j} P_{i}(j) .
$$

Writing $2^{k}=\sum_{i=0}^{n} A_{i}$, we get the following bound.

Proposition 4.1 If $k \geq k_{0}$, then for all $n \geq i \geq 1$ we have

$$
2^{k_{0}} A_{i} \leq \sum_{j=1}^{n} A_{j}\left(\left(\begin{array}{l}
n \\
i
\end{array}\right)-P_{i}(j)\right) .
$$

Proof. We eliminate $B_{i}$ between equation (1) premultiplied by $2^{k}$ and (2) and rearrange. To avoid quadratic terms we bound $2^{k} A_{i}$ below by $2^{k_{0}} A_{i}$. Note that by the generating function for Krawtchouk polynomials $P_{i}(0)=\left(\begin{array}{l}n \\ i\end{array}\right)$.

We consider the block matrix $M\left(n, k_{0}, d\right)$ of order $2 n+d-1$ by $n$ with successive block rows $I_{d-1}, P-\Delta+2^{k_{0}} I_{n},-P$.

Theorem 4.2 If $k \geq k_{0}$, then

$$
2^{L C K[n, d]} \leq 1+U\left(M\left(n, k_{0}, d\right), 0\right)
$$

Proof. The three type of constraints come from, in order, the distance of $C$, the above proposition and the Delsarte inequalities (nonnegativity of the $B_{i}$ 's). 


\begin{tabular}{|c|c|c|c|c|c|c|c|c|c|c|c|c|c|c|c|c|c|c|c|c|c|c|c|c|c|c|c|c|c|c|}
\hline $\mathrm{n} / \mathrm{d}$ & 1 & 2 & 3 & 4 & 5 & 6 & 7 & 8 & 9 & 10 & 11 & 12 & 13 & 14 & 15 & 16 & 17 & 18 & 19 & 20 & 21 & 22 & 23 & 24 & 25 & 26 & 27 & 28 & 29 & 30 \\
\hline 1 & 1 & & & & & & & & & & & & & & & & & & & & & & & & & & & & & \\
\hline 2 & 2 & $0(1)$ & & & & & & & & & & & & & & & & & & & & & & & & & & & & \\
\hline 3 & 3 & 2 & 1 & & & & & & & & & & & & & & & & & & & & & & & & & & & \\
\hline 4 & 4 & $2(3)$ & $\underline{1}$ & $0(1)$ & & & & & & & & & & & & & & & & & & & & & & & & & & \\
\hline 5 & 5 & 4 & 2 & $\underline{1}$ & 1 & & & & & & & & & & & & & & & & & & & & & & & & & \\
\hline 6 & 6 & $4(5)$ & 3 & 2 & $\underline{1}$ & $0(1)$ & & & & & & & & & & & & & & & & & & & & & & & & \\
\hline 7 & 7 & 6 & 4 & 3 & $\underline{1}$ & $\underline{1}$ & 1 & & & & & & & & & & & & & & & & & & & & & & & \\
\hline 8 & 8 & $6(7)$ & $\underline{4}$ & $3(4)$ & 2 & $\underline{1}$ & $\underline{1}$ & $0(1)$ & & & & & & & & & & & & & & & & & & & & & & \\
\hline 9 & 9 & 8 & 5 & 4 & $\underline{2}$ & 2 & $\underline{1}$ & $\underline{1}$ & 1 & & & & & & & & & & & & & & & & & & & & & \\
\hline 10 & 10 & $8(9)$ & 6 & 5 & 3 & 2 & $\underline{1}$ & $\underline{1}$ & $\underline{1}$ & $0(1)$ & & & & & & & & & & & & & & & & & & & & \\
\hline 11 & 11 & 10 & 7 & 6 & 4 & 3 & 2 & $\underline{1}$ & $\underline{1}$ & $\underline{1}$ & 1 & & & & & & & & & & & & & & & & & & & \\
\hline 12 & 12 & $10(11)$ & 8 & 7 & $\underline{5}$ & 4 & $\underline{2}$ & 2 & $\underline{1}$ & $\underline{1}$ & $\underline{1}$ & $0(1)$ & & & & & & & & & & & & & & & & & & \\
\hline 13 & 13 & 12 & 9 & 8 & $6^{*}$ & $5^{*}$ & 3 & 2 & $\underline{1}$ & $\underline{1}$ & $\underline{1}$ & $\underline{1}$ & 1 & & & & & & & & & & & & & & & & & \\
\hline 14 & 14 & $12(13)$ & 10 & 9 & $7^{*}$ & $6^{*}$ & 4 & 3 & 2 & $\underline{1}$ & $\underline{1}$ & $\underline{1}$ & $\underline{1}$ & $0(1)$ & & & & & & & & & & & & & & & & \\
\hline 15 & 15 & 14 & 11 & 10 & $8^{*}$ & $7^{*}$ & 5 & 4 & $\underline{2}$ & 2 & $\underline{1}$ & $\underline{1}$ & $\underline{1}$ & $\underline{1}$ & 1 & & & & & & & & & & & & & & & \\
\hline 16 & 16 & $14(15)$ & $\underline{11}$ & $10(11)$ & 8 & $7\left(8^{*}\right)$ & $\underline{5}$ & $4(5)$ & $\underline{2}$ & 2 & $\underline{1}$ & $\underline{1}$ & $\underline{1}$ & $\underline{1}$ & $\underline{1}$ & $0(1)$ & & & & & & & & & & & & & & \\
\hline 17 & 17 & 16 & 12 & 11 & 9 & 8 & 6 & 5 & 3 & $\underline{2}$ & 2 & $\underline{1}$ & $\underline{1}$ & $\underline{1}$ & $\underline{1}$ & $\underline{1}$ & 1 & & & & & & & & & & & & & \\
\hline 18 & 18 & $16(17)$ & 13 & 12 & $\underline{10}$ & 9 & 7 & 6 & $\underline{4}$ & 3 & $\underline{2}$ & 2 & $\underline{1}$ & $\underline{1}$ & $\underline{1}$ & $\underline{1}$ & $\underline{1}$ & $\left.\begin{array}{lll}0 & 1\end{array}\right)$ & & & & & & & & & & & & \\
\hline 19 & 19 & 18 & 14 & 13 & $11^{*}$ & $10^{*}$ & 8 & 7 & $5^{*}$ & $4^{*}$ & $\underline{2}$ & 2 & $\underline{1}$ & $\underline{1}$ & $\underline{1}$ & $\underline{1}$ & $\underline{1}$ & $\underline{1}$ & 1 & & & & & & & & & & & \\
\hline 20 & 20 & $18(19)$ & 15 & 14 & $12^{*}$ & $11^{*}$ & 9 & 8 & $6^{*}$ & $5^{*}$ & 3 & $\underline{2}$ & 2 & $\underline{1}$ & $\underline{1}$ & $\underline{1}$ & $\underline{1}$ & $\underline{1}$ & $\underline{1}$ & $0(1)$ & & & & & & & & & & \\
\hline 21 & 21 & 20 & 16 & 15 & 12 & $12^{*}$ & 10 & 9 & $\underline{6}$ & $6^{*}$ & $\underline{3}$ & 3 & $\underline{2}$ & 2 & $\underline{1}$ & $\underline{1}$ & $\underline{1}$ & $\underline{1}$ & $\underline{1}$ & $\underline{1}$ & 1 & & & & & & & & & \\
\hline 22 & 22 & $20(21)$ & 17 & 16 & 13 & 12 & 11 & 10 & $7^{*}$ & 6 & 4 & 3 & $\underline{2}$ & 2 & $\underline{1}$ & $\underline{1}$ & $\underline{1}$ & $\underline{1}$ & $\underline{1}$ & $\underline{1}$ & $\underline{1}$ & $0(1)$ & & & & & & & & \\
\hline 23 & 23 & 22 & 18 & 17 & 14 & 13 & 12 & 11 & $8^{*}$ & $7^{*}$ & 5 & 4 & $\underline{2}$ & $\underline{2}$ & 2 & $\underline{1}$ & $\underline{1}$ & $\underline{1}$ & $\underline{1}$ & $\underline{1}$ & $\underline{1}$ & $\underline{1}$ & 1 & & & & & & & \\
\hline 24 & 24 & $22(23)$ & 19 & 18 & $\underline{15}$ & 14 & $\underline{12}$ & $11(12)$ & $\underline{9}$ & $8^{*}$ & $\underline{6}$ & 5 & 3 & $\underline{2}$ & $\underline{2}$ & 2 & $\underline{1}$ & $\underline{1}$ & $\underline{1}$ & $\underline{1}$ & $\underline{1}$ & $\underline{1}$ & $\underline{1}$ & $0(1)$ & & & & & & \\
\hline 25 & 25 & 24 & 20 & 19 & $16^{*}$ & $15^{*}$ & $\underline{13}$ & 12 & $10^{*}$ & 9* & 6 & $6^{*}$ & $\underline{3}$ & 3 & $\underline{2}$ & 2 & $\underline{1}$ & $\underline{1}$ & $\underline{1}$ & $\underline{1}$ & $\underline{1}$ & $\underline{1}$ & $\underline{1}$ & $\underline{1}$ & 1 & & & & & \\
\hline 26 & 26 & $24(25)$ & 21 & 20 & $17^{*}$ & 16 & $14^{*}$ & $13^{*}$ & $10^{*}$ & $10^{*}$ & 7 & 6 & 4 & 3 & $\underline{2}$ & $\underline{2}$ & 2 & $\underline{1}$ & $\underline{1}$ & $\underline{1}$ & $\underline{1}$ & $\underline{1}$ & $\underline{1}$ & $\underline{1}$ & $\underline{1}$ & $0(1)$ & & & & \\
\hline 27 & 27 & 26 & 22 & 21 & $18^{*}$ & $17^{*}$ & 14 & $14^{*}$ & $11^{*}$ & $10^{*}$ & $\underline{8}$ & 7 & 5 & 4 & 3 & $\underline{2}$ & $\underline{2}$ & 2 & $\underline{1}$ & $\underline{1}$ & $\underline{1}$ & $\underline{1}$ & $\underline{1}$ & $\underline{1}$ & $\underline{1}$ & $\underline{1}$ & 1 & & & \\
\hline 28 & $27(28)$ & $26(27)$ & 22 & 21 & $18^{*}$ & $17^{*}$ & 14 & $14^{*}$ & $11^{*}$ & $10^{*}$ & $\underline{8}$ & 7 & 5 & 4 & 3 & $\underline{2}$ & $\underline{2}$ & 2 & $\underline{1}$ & $\underline{1}$ & $\underline{1}$ & $\underline{1}$ & $\underline{1}$ & $\underline{1}$ & $\underline{1}$ & $\underline{1}$ & $\underline{1}$ & $0(1)$ & & \\
\hline 29 & $28(29)$ & 27 & 24 & 23 & $20^{*}$ & $19^{*}$ & $16^{*}$ & $15^{*}$ & $13^{*}$ & $12^{*}$ & $10^{*}$ & 9* & $7^{*}$ & $6^{*}$ & 4 & 3 & $\underline{2}$ & $\underline{2}$ & 2 & $\underline{1}$ & $\underline{1}$ & $\underline{1}$ & $\underline{1}$ & $\underline{1}$ & $\underline{1}$ & $\underline{1}$ & $\underline{1}$ & $\underline{1}$ & 1 & \\
\hline 30 & $29(30)$ & $28(29)$ & 25 & 24 & 20 & $20^{*}$ & $17^{*}$ & $16^{*}$ & $14^{*}$ & $13^{*}$ & 10 & $10^{*}$ & $\underline{7}$ & $7^{*}$ & 5 & 4 & $\underline{2}$ & $\underline{2}$ & $\underline{2}$ & 2 & $\underline{1}$ & $\underline{1}$ & $\underline{1}$ & $\underline{1}$ & $\underline{1}$ & $\underline{1}$ & $\underline{1}$ & $\underline{1}$ & $\underline{1}$ & $0(1)$ \\
\hline
\end{tabular}

Table 1: ( ) : classical LP bound value, if different

$\therefore$ no such code with those parameters

* : larger than the dimension of the best known code with the given parameters 


\section{Constructions}

\subsection{Rings}

In this section, we shall examine a family of rings over which we can define a Gray map which can be used to construct LCD codes.

The ring $R_{k}$ is defined as $R_{k}=\mathbb{F}_{2}\left[u_{1}, u_{2}, \ldots, u_{k}\right] /\left\langle u_{i}^{2}, u_{i} u_{j}-u_{j} u_{i}\right\rangle$. The ring $R_{k}$ has $\left|R_{k}\right|=2^{2^{k}}$ and it is a non-chain ring which has characteristic 2 with maximal ideal $\mathbf{M}=\left\langle u_{1}, u_{2}, \ldots, u_{k}\right\rangle$ and $\operatorname{Soc}\left(R_{k}\right)=\left\langle u_{1} u_{2} \cdots u_{k}\right\rangle$.

We can now construct a linear Gray map from $R_{k}$ to $\mathbb{F}_{2}^{2^{k}}$. Let $\phi_{1}$ be the map defined on $R_{1}$, namely $\phi_{1}(a+b u)=(b, a+b)$. Then let $c \in R$. We can write $c=c_{1}+u_{k} c_{2}$ where $c_{1}, c_{2}$ are elements of the ring $R_{k-1}$ of order $2^{2^{k-1}}$, then we define

$$
\phi_{k}(c)=\left(\phi_{k-1}\left(c_{2}\right), \phi_{k-1}\left(c_{1}\right)+\phi_{k-1}\left(c_{2}\right)\right) .
$$

The map $\phi_{k}$ is a weight preserving map which we then expand coordinatewise to $R^{n}$.

The following can be found in [4].

Lemma 5.1 The map $\phi_{k}: R_{k} \rightarrow \mathbb{F}_{2}^{2^{k}}$ is a linear bijection. Moreover, we have $\phi\left(C^{\perp}\right)=\phi(C)^{\perp}$.

We can define an LCD code over $R_{k}$ in the usual way by saying that the code is LCD if its intersection with its dual is $\{0\}$. This leads immediately to the following.

Theorem 5.2 Let $C$ be a LCD code of length $n$ over $R_{k}$ then $\phi(C)$ is a binary LCD code of length $2^{k} n$.

Proof. We have that $\phi(C) \cap \phi(C)^{\perp}=\phi(C) \cap \phi\left(C^{\perp}\right)$. Then since $\phi$ is a bijection and $C \cap C^{\perp}=0$ we have the desired result.

Theorem 5.3 There are no non-trivial LCD codes of length 1 over $R_{k}$.

Proof. Any code of length 1 is an ideal in the ring $R$ and hence $C$ and $C^{\perp}$ are ideals and hence both contained in the maximal ideal $M$. This implies their intersection contains $\mathbf{M}^{\perp}=S o c(R)=\left\{\mathbf{0}, u_{1} u_{2} \cdots u_{k}\right\}$ which is non-trivial.

Theorem 5.4 (i) Let $G$ be a binary matrix such that $G G^{T}=I_{n}$, then $G$ generates an LCD code $C$ of length $n$ over $R_{k}$ and $\phi_{k}(C)$ is a binary LCD code of length $2^{k} n$. 
(ii) Let $G$ be a binary matrix such that $G G^{T}=J_{n}-I_{n}$, n even, then $G$ generates an LCD code $C$ of length $n$ over $R_{k}$ and $\phi_{k}(C)$ is a binary LCD code of length $2^{k} n$.

Proof. The first item follows from Corollary 2.2 followed by Theorem 5.2, The second item follows from Lemma 2.4 followed by Theorem 5.2 .

\subsection{Orthogonal matrices}

One way to construct generator matrices $G$ such that $G G^{T}$ is invertible is to demand $G G^{T}=I$. Such rectangular matrices $G$ can be obtained as row submatrices from so-called orthogonal matrices over $\mathbb{F}_{2}$. Define the orthogonal group $O(n, 2)$ as the set of all matrices $X$ of $G L(n, 2)$ satisfying $X X^{T}=I_{n}$. The order of this group is known to be

$$
|O(n, 2)|=2^{k^{2}} \prod_{i=1}^{k}\left(2^{2 i}-1\right),
$$

where $k=\lfloor n / 2\rfloor$. See [15]. Generators for this group are as follows. Let $P_{n}$ denote the matrix group of permutation matrices of order $n$. A transvection attached to vector $u$ is a transform $T_{u}$ that maps all $x \in \mathbb{F}_{2}^{n}$ to $T_{u}(x)=x+(x, u) u$. By [14, Th. 19] we know that for $n \geq 4$ we have $O(n, 2)=\left\langle P_{n}, T_{u}\right\rangle$, for any $u$ of Hamming weight 4 . Since, as is well-known, is generated by a transposition and an $n$-cycle the group $O(n, 2)$ is generated by three generators for $n \geq 4$. It is therefore easy to generate random orthogonal matrices of order $n$ for large $n$. This technique was used in [12, 13] in the contexts of self-dual codes and self-dual Boolean functions, respectively.

Another technique is to use the correspondence with systematic generator matrices of self-dual codes. Thus $(I, X)$ is self-dual if and only if $X \in G L(n, 2)$.

Example: The Golay code of length 24 gives an orthogonal matrix of order 12, which, in turn, by taking the span of some rows gives LCD codes with parameters $[12,6,3],[12,4,4],[12,8,2]$.

\subsection{Block designs}

Recall that for a Balanced Incomplete Block Design (BIBD) with parameters $(b, v, k, r, \lambda)$, the $b$ indicates the size of the blocks, $v$ indicates the number of varieties, $k$ indicates the number of varieties on a block, $r$ indicates the number of blocks through a variety and through any 2 varieties there are $\lambda$ blocks. We refer to this $\mathrm{BIBD}$ as a $2-(v, k, \lambda)$ design. 
Theorem 5.5 Denote by $Q$ the variety vs block incidence matrix of a $2-(v, k, \lambda)$ BIBD. If $r k(r-\lambda) \neq 0(\bmod 2)$ then $Q$ generates an LCD code.

Proof. It is well-known, [1, Th. 1.4.1], that

$$
\operatorname{det}\left(Q Q^{T}\right)=r k(r-\lambda)^{v-1} .
$$

Thus, provided that $r k(r-\lambda) \neq 0 \quad(\bmod 2)$, we see that the row span of $Q$ is LCD of parameters $[b, v, \geq 2(r-\lambda)]$ by Corollary 2.3 .

\subsection{Table of lower bounds on $\operatorname{LCK}[n, d]$}

The first seven rows of the following table were filled up using the codes in Section 3.2 and the results in the following section. The remaining rows of the following table were filled up using orthogonal matrices constructed from

- extended quadratic residue codes for $n=12,16,24$;

- database of self-dual codes [6] for the other $n=8,9,10,11,13,14,15,18,20$;

- group generation as in $\S 5.2$ for $n=17,19,21,22,23$. 


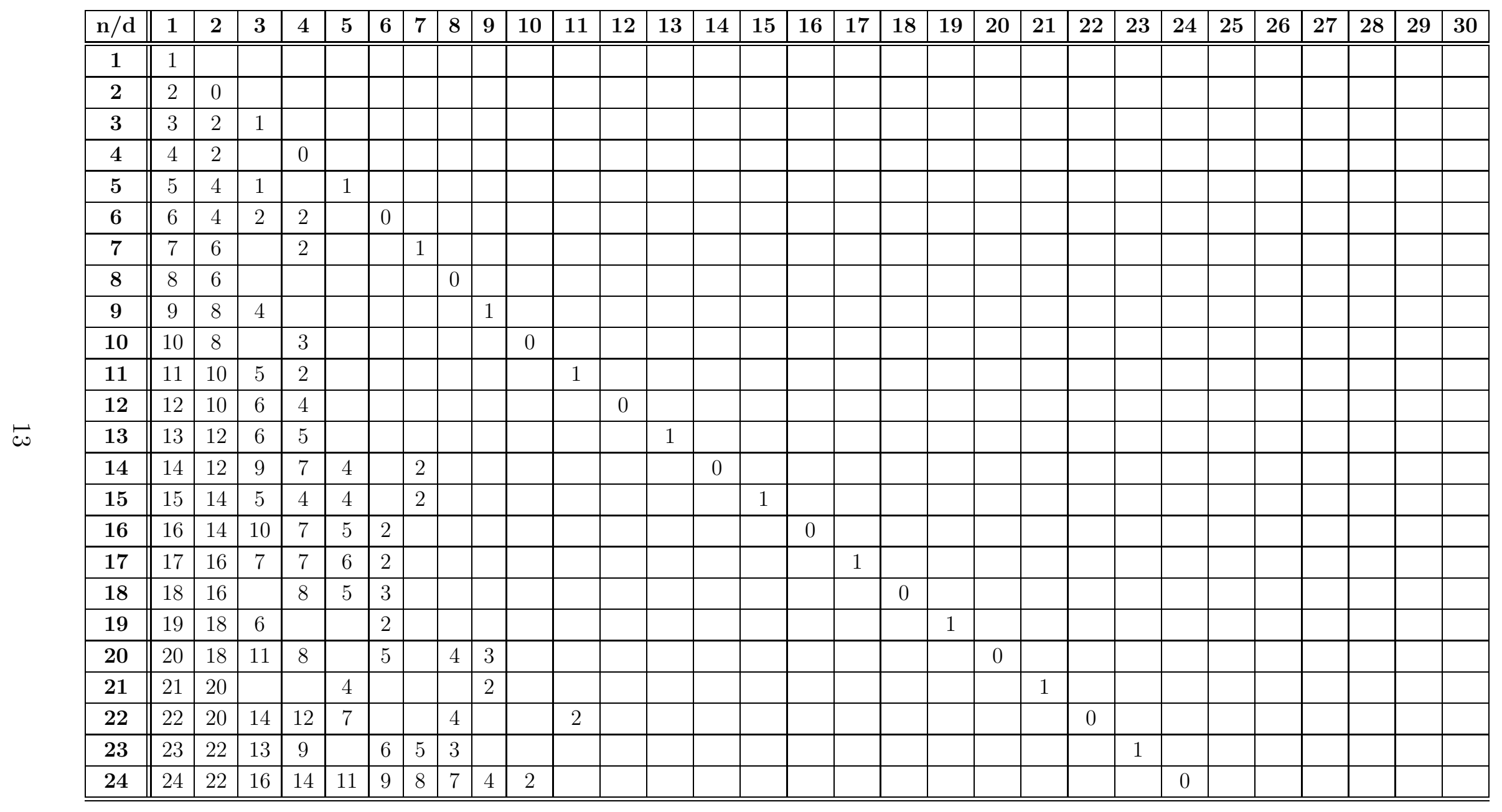

Table 2: Lower bounds on $\operatorname{LCK}[n, d]$ 


\section{Conclusion and open problems}

This paper is dedicated to LCD codes. A linear programming bound on the largest size of an LCD code of given length and distance was derived. It is a worthwhile project to derive an asymptotic version of that bound. More generally semi-definite programming bounds are worth exploring. A construction based on orthogonal matrices was derived. It would be interesting to see other classes of combinatorial matrices enter the problem. Improving the table of lower bounds by using codes over rings or symmetric designs is also worth considering.

\section{References}

[1] E.F. Assmus Jr, J.D. Key, Designs and their codes, Cambridge TM 103, Cambridge (1992).

[2] A. Bonisoli, Every equidistant linear code is a sequence of dual Hamming codes, Ars Combinatoria, 18 (1983), 181-186.

[3] C. Carlet and S. Guilley, "Complementary dual codes for counter-measures to side-channel attacks", Proceedings of the 4th ICMCTA Meeting, Palmela, Portugal, 2014.

[4] S.T. Dougherty, B. Yildiz, S. Karadeniz, Codes over $R_{k}$, Gray maps and their Binary Images, Finite Fields and their Applications, Volume 17, Number 3, (2011) 205-219.

[5] M. Grassl, "Bounds on the minimum distance of linear codes and quantum codes," Online available at http://www.codetables.de. Accessed on 201412-03.

[6] M. Harada, A. Munemasa, http://www.math.is.tohoku.ac.jp/ munemasa/research/codes/data/2/

[7] W.C. Huffman and V.S. Pless, Fundamentals of Error-correcting Codes, Cambridge: Cambridge University Press, (2003).

[8] F.J. MacWilliams, N.J.A. Sloane, The theory of error correcting codes, (1981) North Holland.

[9] J.L. Massey, Linear codes with complementary duals, Discrete Mathematics, 106 - 107, 337-342, 1992. 
[10] E.Rains and N.J.A. Sloane, Self-dual codes, in Handbook of Coding Theory, V.S. Pless and W.C. Huffman, eds., Elsevier, Amsterdam, (1998), 177-294.

[11] H. Van Tilborg, On the uniqueness resp. non existence of certain codes meeting the Griesmer bound, Information and Contr. 44 (1980) 16-35.

[12] C. Aguilar-Melchor, P. Gaborit, J-L. Kim, L. Sok, P. Solé, classification of extremal and s-extremal binary self-dual codes of length 38. IEEE Trans. on Information Theory IT-58 (2012) 2253-2252.

[13] T. Feulner,L. Sok, P. Solé, A. Wassermann, The classification of self dual bent functions in eight variables, Designs, Codes, and Cryptography 68 (2013) 395-406.

[14] G. Janusz, Parametrization of self-dual codes by orthogonal matrices, Finite Fields and their Appl. 13 (2007) 450-491.

[15] F. J. MacWilliams, Orthogonal matrices over finite fields, American Math Monthly Vol. 76, No. 2 (1969), 152-164.

[16] V. Pless, Introduction to the theory of error-correcting codes, Wiley (1998).

[17] H. N. Ward, Divisibility of codes meeting the Griesmer bound. J. Combin. Theory Ser. A 83 , no. 1, (1998) 79-93. 